\title{
FRMD4A: A potential therapeutic target for the treatment of tongue squamous cell carcinoma
}

\author{
XIANGHUAI ZHENG ${ }^{*}$, BO JIA*, XI LIN, JIUSONG HAN, XIAOLING QIU, HONGXING CHU, \\ XIANG SUN, WEITAO HU, JIE PAN, JUN CHEN and JIANJIANG ZHAO \\ Laboratory for Oral Diseases, Department of Oral Surgery, Guangdong Provincial Stomatological Hospital, \\ Southern Medical University, Guangzhou, Guangdong 510280, P.R. China
}

Received December 12, 2015; Accepted July 14, 2016

DOI: $10.3892 /$ ijmm.2016.2745

\begin{abstract}
The aim of the present study was to identify agents capable of inhibiting the invasion and metastasis of tongue squamous cell carcinoma and thereby improve the outcomes of patients suffering from tongue cancer. FRMD4A antibodies were used to probe 78 paraffin-embedded specimens of tongue squamous cell carcinoma and 15 normal tongue tissues, which served as controls. Immunohistochemical methods were then used for analysis. Clinical pathological parameters were obtained, and the association between FRMD4A expression in the samples and the pathological parameters was analyzed. The human tongue cancer cell line CAL27 was used to study the effects of FRMD4A. CAL27 cells were transfected with small-interfering RNA against FRMD4A (FRMD4A-siRNA) and the mRNA and protein levels of FMRD4A were then evaluated by RT-qPCR and western blot analysis, respectively. The proliferation and cell-cycle assays of CAL27 cells were evaluated using the CCK8 method and flow cytometry. The invasion and migration of the cells were measured using a Matrigel invasion chamber and a scratch assay, respectively. The results showed FRMD4A overexpression in tongue squamous cell carcinoma, and the positive reaction was predominately located in the cytoplasm. Tumor clinical stage and lymph node metastasis showed a statistically significant correlation with FRMD4A expression. Transient silencing of the FRMD4A gene for 24 and $48 \mathrm{~h}$ significantly decreased the mRNA and protein expression of FRMD4A, respctively. Silencing FRMD4A gene reduced the proliferation of CAL27
\end{abstract}

Correspondence to: Professor Jianjiang Zhao, Laboratory for Oral Diseases, Department of Oral Surgery, Guangdong Provincial Stomatological Hospital, Southern Medical University, 366 South Jiang Nan Road, Haizhu, Guangzhou, Guangdong 510280, P.R. China E-mail: zjj2521@sina.com

\section{${ }^{*}$ Contributed equally}

Key words: tongue squamous cell carcinoma, FRMD4A, CAL27, gene silencing cells and led to cell cycle arrest in the G1 phase, as well as significantly suppressing the migration and invasion capacity of CAL27 cells. The findings of the present study suggest that FRMD4A expression correlates with the development of tongue squamous cell carcinoma. For this reason, FRMD4A merits further study as it may be suitable for use as a therapeutic agent in antitumor treatment regimens.

\section{Introduction}

Oral squamous cell carcinoma (OSCC) contributes to $90 \%$ of oral malignant tumors $(1,2)$. Most cases of OSCC are treated through surgery or radiation therapy, with or without concomitant chemotherapy. The overall five-year survival rate remains $<50 \%$ even though there have been great advances in surgical techniques and general patient care $(3,4)$. The presence of cervical lymph node metastasis causes the high mortality of OSCC. For this reason, many studies have been devised to identify the molecular markers capable of inhibiting tumor invasion and metastasis in order to improve treatment outcomes for patients suffering from OSCC.

FERM domain containing 4A (FRMD4A) belongs to the FERM superfamily of proteins, which are ubiquitous components of the cytocortex and are involved in cell transport, cell structure and signaling functions. Proteins in the FERM family have been shown to participate in many cellular events, including tumor progression and metastasis. These proteins act as regulators or scaffolding units and they are involved in the functions of many membrane-associated factors $(5,6)$. Head and neck carcinomas are particularly affected by FRMD4A protein activity. Head and neck squamous cell carcinomas are associated with a poor prognosis when there is high expression of FRMD4A protein, and FRMD4A has been shown to promote the growth of cancer cells in these types of carcinomas (7).

Previous studies have indicated that FRMD4A may play a crucial role in tongue squamous cell carcinoma. In the present study, the association between FRMD4A expression and clinicopathological parameters was analyzed through immunohistochemistry. The effects of FRMD4A on cell invasion and migration were examined in a tongue cancer cell line transfected with small-interfering RNA against FRMD4A (FRMD4A-siRNA). The effects of FRMD4A in tongue squamous cell carcinoma were also explored. 


\section{Materials and methods}

Ethics statement. Signed informed consent was obtained from all participants prior to participation in the study. The results of the study were collected anonymously. The study was approved by the Human Ethics Committee of Guangdong Provincial Stomatological Hospital (Guangzhou, China). The use of participants' thumbprints was also approved by this committee.

Participants. This investigation was designed as a retrospective study. All participants underwent surgery at the Department of Oral and Maxillofacial Surgery of the Guangdong Provincial Stomatological Hospital between 2012 and 2013. For these patients, we also had access to clinical follow-up data as well as tissue specimens which were obtained from the local pathology repository. All of the tumors were reclassified using hematoxylin and eosin (H\&E)-stained slides. Standard diagnostic criteria was used by a pathologist in order to reassess tumor grade and histological type. The clinical data were obtained by contacting the physicians in charge and reviewing the charts. The histological classification of tumors was based on the WHO classification system.

Immunohistochemistry. FRMD4A expression was determined by immunohistochemichal analysis using a goat polyclonal antibody to detect the recombinant human FRMD4A at a dilution of 1:100 (Abcam, Cambridge, MA, USA). Seventy-eight tongue cancer samples with various levels of FRMD4A expression served as positive and negative controls. Moreover, 15 samples of normal tongue tissue were collected from patients undergoing tongue surgery at Guangdong Provincial Stomatological Hospital. Additionally 15 samples of tongue tissue with leukoplakia were collected. Two observers examined the expression of FRMD4A antigen independently in blinded fashion. Inconsistent cases were reexamined using a double-headed microscope (DM 2500; Leica, Mannheim, Germany) until the observers agreed. FRMD4A expression was defined as the presence of particular staining of the tumor cell surface membranes. By calculating a total immunostaining score as the result of a proportion score and intensity score, each tissue sample was examined for the expression of FRMD4A. The estimated fraction of positive-stained tumor cells was defined as the proportion score ( 0 , none; $1,10 \% ; 2,1-50 \% ; 3,1-80 \% ; 4,>80 \%)$, whereas the estimated staining intensity was demonstrated as the intensity score ( 0 , no staining; 1 , weak; 2 , moderate; 3 , strong) with the aggregate score ranging from 0 to 12 . Overexpression of FRMD4A was defined as an aggregate score of $>4$ as described previously (8).

Culture of cells and siRNA transfection. The human tongue cancer cell line CAL27 was obtained from the Shanghai Ninth People's Hospital (Shanghai, China) and cultivated in Dulbecco's modified Eagle's medium (DMEM) (Invitrogen, Carlsbad, CA, USA) supplemented with $100 \mathrm{U} / \mathrm{ml}$ penicillin and the same dose of streptomycin (Gibco, Grand Island, NY, USA) and 10\% (v/v) fetal bovine serum (HyClone, Logan, UT, USA). The cells were then incubated in an incubator with $5 \% \mathrm{CO}_{2}$ at a temperature of $37^{\circ} \mathrm{C}$. GenePharma Co., Ltd.
(Shanghai, China) provided the FRMD4A-siRNA (5'-GCCAUUCAGUCCCAGAUUATT-3') and control-siRNA (5'-UUCUCCGAACGUGUCACGUTT-3'). Lipofectamine RNAiMAX reagent (Invitrogen) was used for transient transfection, according to the manufacturer's instructions.

RNA isolation and reverse transcription-quantitative polymerase chain reaction (RT-qPCR). TRIzol reagent (Invitrogen) was used to isolate the total RNA, according to the manufacturer's instructions. Template cDNA was synthesized from whole RNA (1 $\mu \mathrm{g})$ with a Quantscript RT kit (Tiangen, Beijing, China) using random primers and a ribonuclease inhibitor. cDNA ( $1 \mu \mathrm{l}$ from the sample) was integrated with $19 \mu \mathrm{l}$ of the master reaction mix. The 40 cycles of RT-qPCR included denaturation at $95^{\circ} \mathrm{C}$ for $15 \mathrm{sec}$, annealing at $60^{\circ} \mathrm{C}$ for $32 \mathrm{sec}$ and extension at $60^{\circ} \mathrm{C}$ for $1 \mathrm{~min}$. A Real Master Mix kit (SYBR-Green) and 7500 Real-Time PCR system were used (both from Applied Biosystems, Foster City, CA, USA). The following primer sequences were used in the reaction: FRMD4A sense, 5'-CCATTCTGGATGGGTC TGA and antisense, 5'-AGGGAGGAATCCAGGAAACA; GAPDH sense, 5'-GGGAAACTGTGGCGTGAT and antisense, 5'-GAGTGGGTGTCGCTGTTGA. Each experiment was performed three times.

Western blot analysis. Cells were harvested after $48 \mathrm{~h}$ of treatment with FRMD4A-siRNA and NC-siRNA. Standard procedures for western blot analysis were used. Briefly, CAL27 cells were washed with ice-cold phosphate-buffered saline (PBS), scraped and then homogenized in RIPA buffer. Centrifugation was used to remove the cell debris and the supernatant was quickly frozen. A standard bicinchonic acid (BCA) method was performed to quantify the proteins in the supernatants. A $15 \%$ sodium dodecyl sulfate-polyacrylamide gel electrophoresis (SDS-PAGE) procedure was used to separate the proteins, which were then transferred to a PVDF membrane. The membrane was blocked with $5 \%$ bovine serum albumin (BSA), according to the manufacturer's instructions, for $2 \mathrm{~h}$. The membrane was then incubated with special antibodies that had been designed to detect the selected proteins [FRMD4A(G-18), sc-242858; Santa Cruz Biotechnology, Santa Cruz, CA, USA]. A LAS3000 ${ }^{\circledR}$ Luminescent image analyzer was used for visualization and Multi Gauge V3.0 software (Fujifilm, Tokyo, Japan) was used to evaluate protein expression.

Cell proliferation assay. Cell proliferation was detected using the cell counting kit 8 (CCK-8; Dojindo, Kumamoto, Japan) method according to the manufacturer's instructions. Cells were seeded $\left(1 \times 10^{4}\right.$ cells $\left./ \mathrm{ml}\right)$ on 96-well plates and cultured in $5 \% \mathrm{CO}_{2}$ at $37^{\circ} \mathrm{C}$ for $24 \mathrm{~h}$. Transfection was performed when the cells were $30-50 \%$ confluent. Following transfection, $10 \mu \mathrm{l} /$ well of CCK8 was added to the cells and reacted for $4 \mathrm{~h}$. Then, a microplate reader (Bio-Rad, Hercules, CA, USA) was used to calculate the cell proliferative capability by quantifying the light absorbance at $450 \mathrm{~nm}$. Measurements were performed in triplicate.

Cell cycle assay. Propidium iodide (PI) staining and flow cytometry were used to analyze the cell cycles. Approximately $5 \times 10^{5}$ cells were obtained for each group after being harvested for $24 \mathrm{~h}$ 
after transfection. The cells were washed twice with cold PBS and then resuspended in $70 \%$ pre-cooled ethanol. They were then fixed overnight at $4^{\circ} \mathrm{C}$. The fixed cells were washed with PBS and then incubated with RNase A and PI in succession at $37^{\circ} \mathrm{C}$ for $30 \mathrm{~min}$. Fluorescence-activated cell sorting (FACS) analysis was performed and each experiment was performed three times.

Scratch wound-healing assay. A scratch wound-healing assay was performed in order to detect cell migration. Cancer cells were seeded on 24-well plates. The bottom of the well was scratched with the fine end of a pipette tip. The plates were washed twice with PBS in order to remove the detached cells. The remaining adherent cells were then cultured in complete growth medium. After $48 \mathrm{~h}$, a bright-field microscope (BX41; Olympus, Tokyo, Japan) was used to evaluate the extent of space filling by cell migration. The experiments were performed in triplicate.

Matrigel invasion assay. The Corning Matrigel Invasion Chamber technique (Corning, Inc., Acton, MA, USA) was performed for the cell invasion experiment. Cancer cells were collected after $48 \mathrm{~h}$ of transfection. Homogeneous single cell suspensions in the serum-free DMEM were added to the upper chamber at a density of $5 \times 10^{4}$ cells/well. Cells were allowed to invade for $24 \mathrm{~h}$ at $37^{\circ} \mathrm{C}$ in an incubator. The non-invading cells on the upper side of the polyethylene terephthalate (PET) membrane (BD Biosciences, Franklin Lakes, NJ, USA) were removed using cotton swabs. The membrane containing the invading cells was stained with H\&E (Yeasen, Shanghai, China) and fixed with methanol. The numbers of invading cells were detected by light microscopy (BX53M; Olympus).

Statistical analysis. The Statistical Package for the Social Sciences (SPSS) software version 13.0 (SPSS Inc., Chicago, IL, USA) was used to perform statistical analysis. Correlations between FRMD4A expression and clinicopathological variables were analyzed with a $\chi^{2}$-test. Either a one-way ANOVA or an independent samples test was used to analyze the differences between groups. A P-value $<0.05$ was considered to indicate a statistically significant difference.

\section{Results}

Immunohistochemistry. The results of immunohistochemical analysis showed that positive reactions to FRMD4A protein were mainly located in the cytoplasm, and appeared brownish, yellow and granular (Fig. 1). Staining was present mainly in the basal layer and as the degree of tumor malignancy increased, it tended to spread to the stratum spinosum. There was significantly more FRMD4A protein expression in the tongue squamous cancer tissues $(50 / 78,64.1 \%)$ than in the leukoplakia group $(5 / 15,33.3 \%)$ and that of the normal tissue group $(2 / 15,13.3 \%)$ (data not shown). However, the difference between leukoplakia group and the adjacent normal tissue group was not statistically significant.

Clinicopathological features. The association between clinicopathological variables and FRMD4A overexpression is demonstrated in Table I. $\chi^{2}$-testing showed that there was a significant association between FRMD4A overexpression
Table I. Clinicopathological variables and FRMD4A expression in OSCC.

\begin{tabular}{|c|c|c|c|c|c|}
\hline \multirow[b]{2}{*}{ Characteristics } & \multirow[b]{2}{*}{ Patients } & \multicolumn{2}{|l|}{ FRMD4A } & \multirow[b]{2}{*}{$\chi^{2}$} & \multirow[b]{2}{*}{ P-value } \\
\hline & & Overexpression & $\%$ & & \\
\hline \multicolumn{6}{|l|}{ Gender } \\
\hline Male & 40 & 27 & 67.5 & 0.412 & 0.521 \\
\hline Female & 38 & 23 & 60.5 & & \\
\hline \multicolumn{6}{|l|}{ Age } \\
\hline$<55$ & 36 & 21 & 58.3 & 0.967 & 0.325 \\
\hline$\geq 55$ & 42 & 29 & 69.0 & & \\
\hline \multicolumn{6}{|l|}{ Grading } \\
\hline I & 20 & 11 & 55.0 & 1.140 & 0.565 \\
\hline II & 47 & 31 & 66.0 & & \\
\hline III & 11 & 8 & 72.7 & & \\
\hline \multicolumn{6}{|l|}{$\begin{array}{l}\text { Lymph node } \\
\text { metastasis }\end{array}$} \\
\hline Yes & 35 & 28 & 80.0 & 6.973 & 0.008 \\
\hline No & 43 & 22 & 51.2 & & \\
\hline \multicolumn{6}{|l|}{ Clinical stage } \\
\hline $\mathrm{I}+\mathrm{II}$ & 31 & 14 & 45.2 & 8.021 & 0.005 \\
\hline III+IV & 47 & 36 & 76.6 & & \\
\hline
\end{tabular}

OSCC, oral squamous cell carcinoma; FRMD4A, FERM domain containing 4A.

and the presence of lymph node metastases $(\mathrm{P}=0.008)$ as well as the clinical stage $(\mathrm{P}=0.005)$. There was no association between FRMD4A overexpression and gender, age or histologic grading.

Silencing FRMD4A inhibits the mRNA and protein expression of FRMD4A in CAL27 cells. In this experiment, siRNA was used to silence FRMD4A in CAL27 cells. Significant inhibition of FRMD4A in the experimental group was detected 24 and $48 \mathrm{~h}$ after transient transfection in the mRNA $\left(\mathrm{P}_{\mathrm{CAL} 27}=0.02\right)$ and protein $\left(\mathrm{P}_{\mathrm{CAL} 27}=0.03\right)$ levels, respectively. However, there was no significant difference between the untreated group and the control group (Fig. 2).

Silencing FRMD4A reduces the proliferation of CAL27 cells. After addition of CCK8, cell proliferation was checked at 24, 48 and 72 h. As shown in Fig. 3, compared with the control group at 24 and $48 \mathrm{~h}$, the proliferation of CAL27 cells markedly decreases following transfection with FRMD4A-siRNA. However, at $72 \mathrm{~h}$ after transfection, with the weakening effect of RNA interference, the differences between the experimental group and the control groups were not significant.

Silencing FRMD4A leads to cell cycle arrest in CAL27 cells in vitro. FACS analysis revealed that a higher percentage of the cell population was residing in the G1 phase after transfection with FRMD4A-siRNA, indicating that cell cycle progression from the $\mathrm{G} 1$ phase to the $\mathrm{G} 2+\mathrm{S}$ phase was impeded by FRMD4A (Fig. 4). In other words, CAL27 cell proliferation was inhibited. 


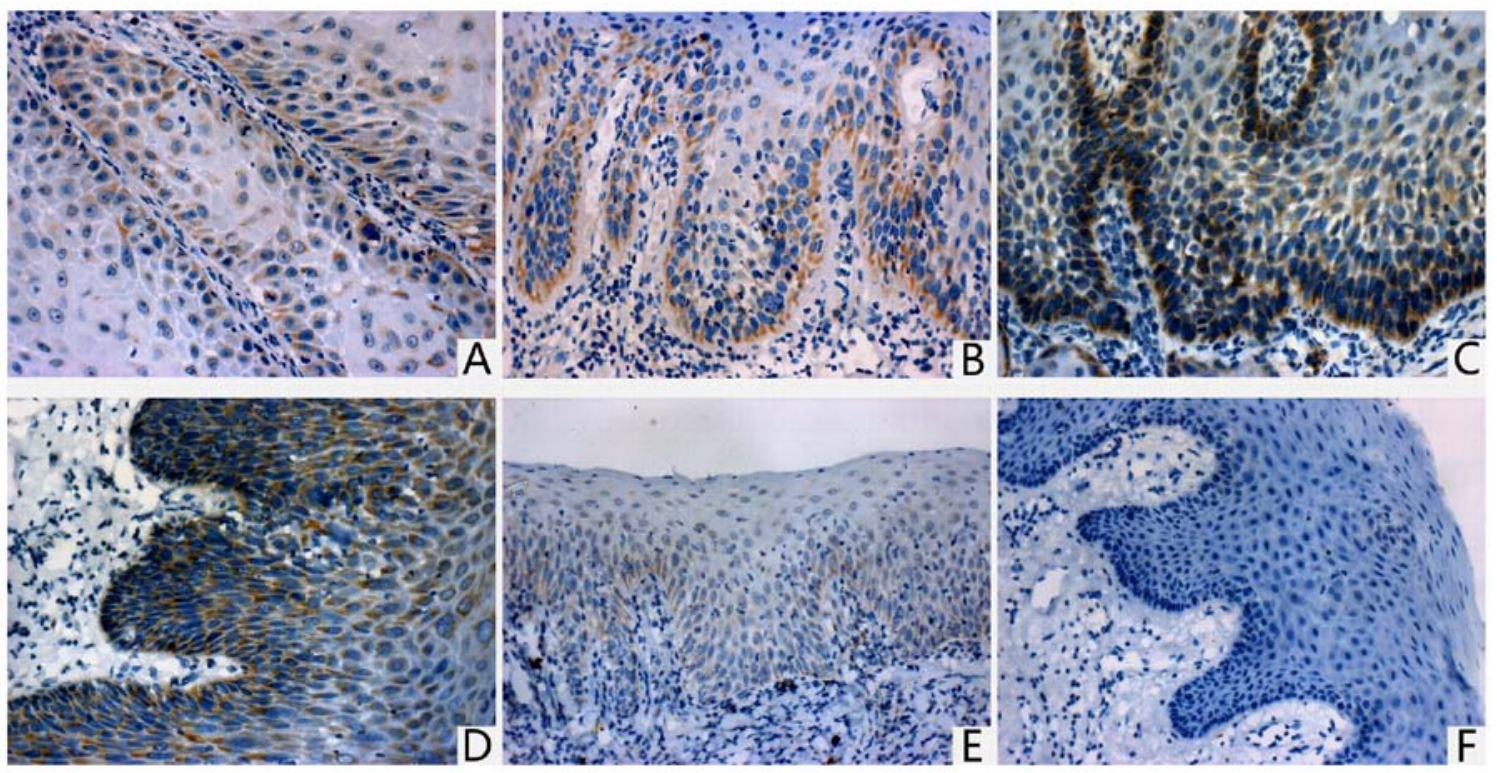

Figure 1. Immunohistochemical staining of FERM domain containing 4A (FRMD4A) expression in oral squamous cell carcinoma (OSCC). Original magnification, $\mathrm{x} 400$. Representative images of (A) well-differentiated (score 2), (B) moderately differentiated (score 6), (C) poorly differentiated (score 12), (D) lymph node metastasis (score 9), (E) leukoplakia (score 3) and (F) normal tongue tissues (score 0). The estimated fraction of positive-stained tumor cells was defined as the proportion score $(0$, none; $1,10 \% ; 2,1-50 \% ; 3,1-80 \% ; 4,>80 \%)$, whereas the estimated staining intensity was demonstrated as the intensity score $(0$, no staining; 1 , weak; 2 , moderate; 3 , strong) with the aggregate score ranging from 0 to 12 . Overexpression of FRMD4A was defined as an aggregate score of $>4$.
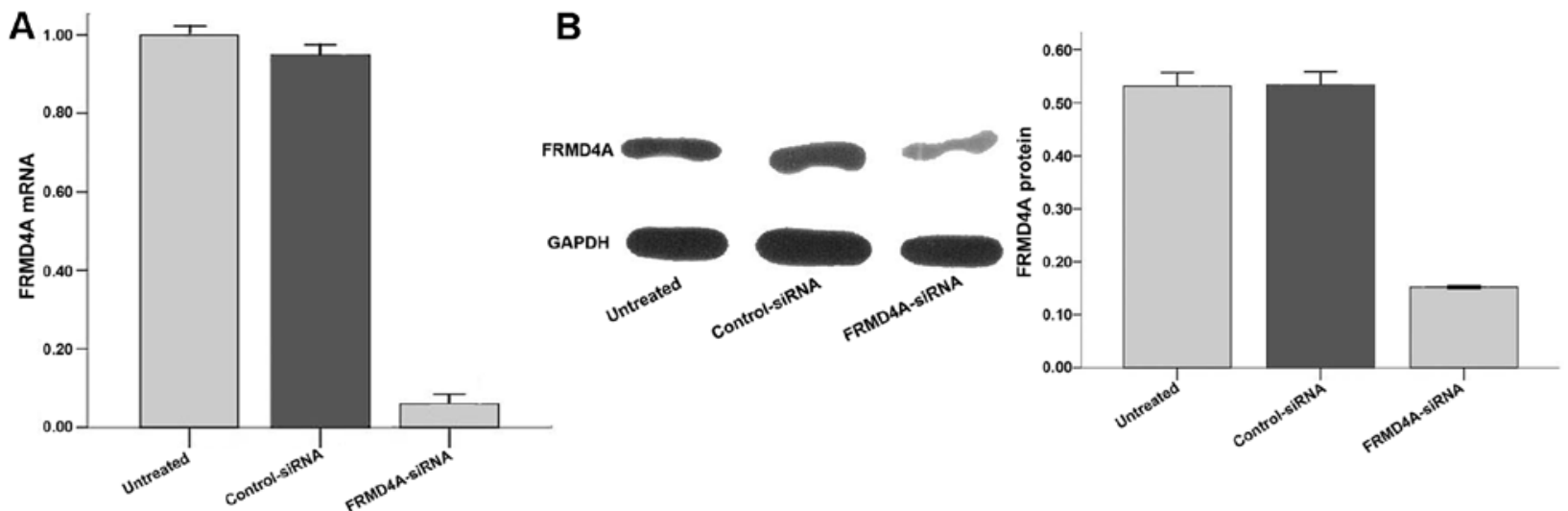

Figure 2. Comparison of the mRNA and protein levels of FERM domain containing 4A (FRMD4A) in CAL27 cells. The expression of FRMD4A was clearly inhibited by silencing FRMD4A. (A) Quantitative analysis of the mRNA levels of FRMD4A evaluated by RT-qPCR after $24 \mathrm{~h}$. (B) Representative western blot and quantitative anlysis of protein expression of FRMD4A evaluated after $48 \mathrm{~h}$.

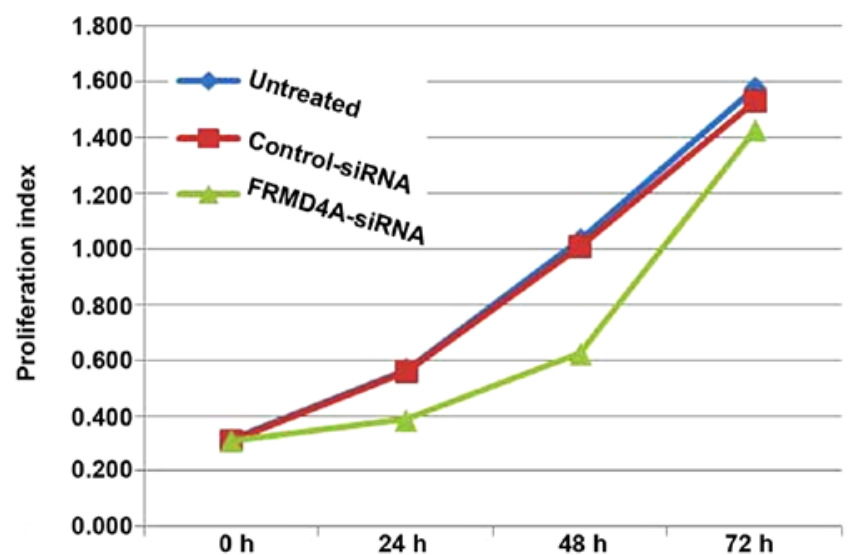

Figure 3. Analysis of CAL27 cell proliferation. At 24 and $48 \mathrm{~h}$ after transfection with FERM domain containing 4A (FRMD4A)-siRNA, cell proliferation was found to be inhibited in the experimental group.
Silencing FRMD4A decreases migration and invasion of CAL27 cells. The migration capacity of FRMD4A-siRNA transfected cells was lower than in the other control group (Fig. 5). FRMD4A-silenced CAL27s cells required more time for space closure than the control cells. The invasion capacity of CAL27 cells $\left(\mathrm{P}_{\mathrm{CAL} 27}=0.01\right)$ was reduced by FRMD4A siRNA treatment. Fewer FRMD4A-siRNA-treated cells migrated on the PET membrane than in the control groups (Fig. 6). The results suggest that FRMD4A modulates the migration and invasion of CAL27 cells in vitro.

\section{Discussion}

The recently discovered FRMD4A gene belongs to the FERM superfamily of proteins and it is located on human chromosome 10p13. The FERM family of proteins link 

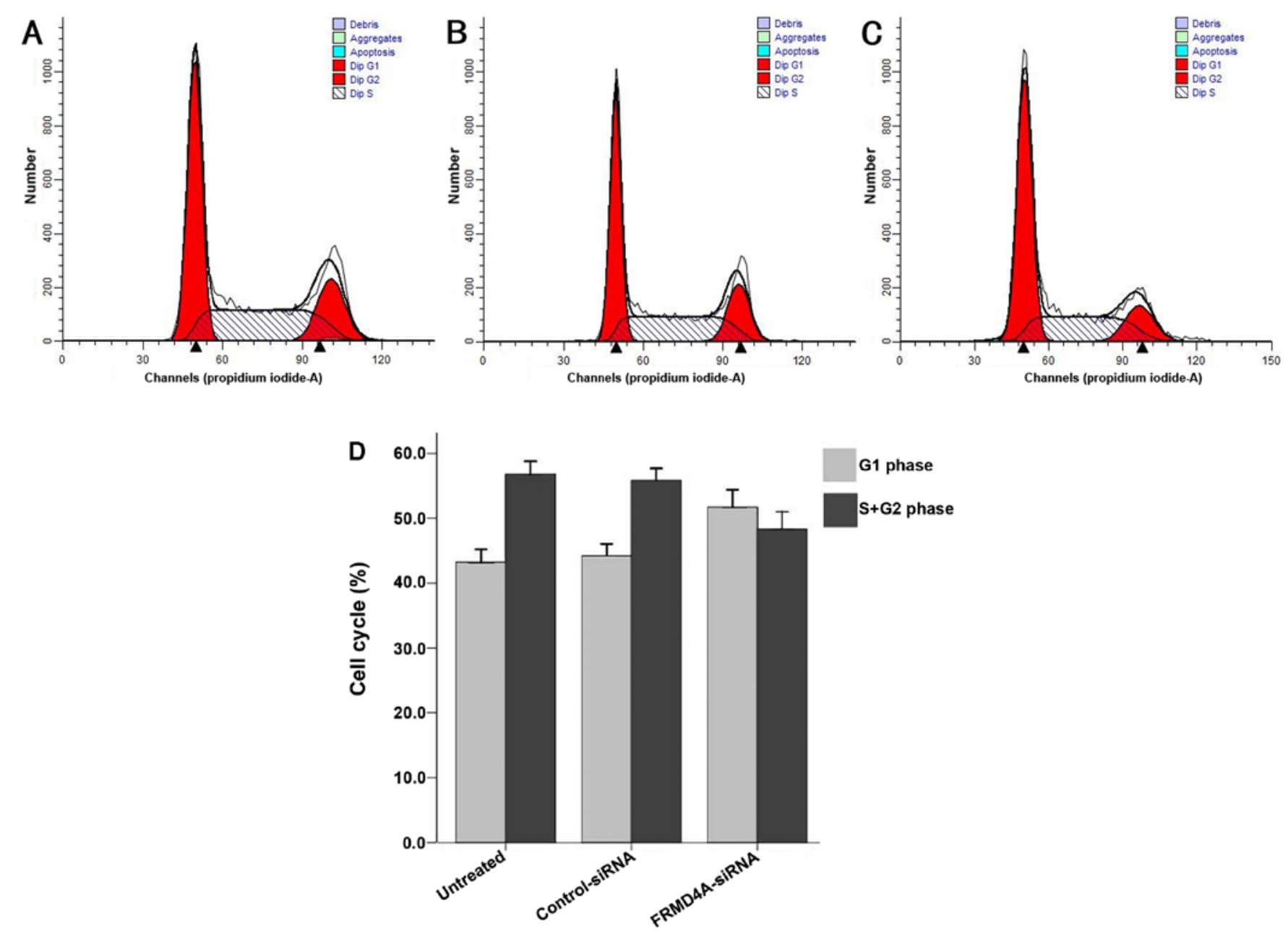

Figure 4. Effect of silencing FERM domain containing 4A (FRMD4A) on the cell cycle in CAL27 cells. Fluorescence-activated cell sorting (FACS) analysis revealed that there was a significantly higher percentage of cells in the G1 phase in the experimental group transfected with FRMD4A-siRNA (C) than in the control groups which were either (A) untreated or (B) transfected with control-siRNA. (D) The results of FACS analysis are presented in a bar chart.
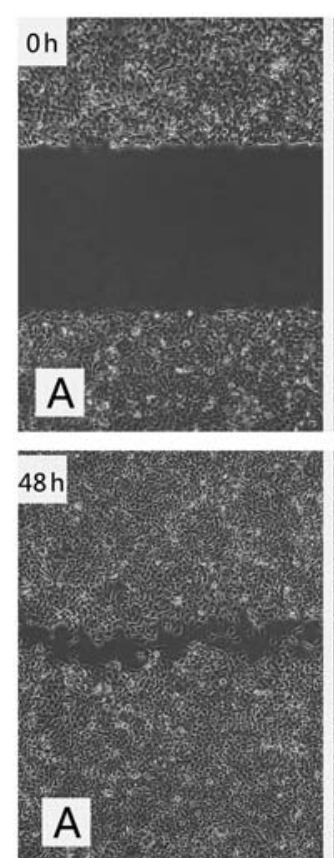
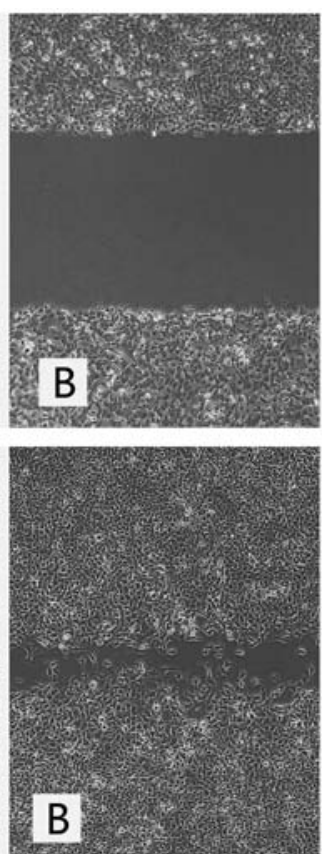
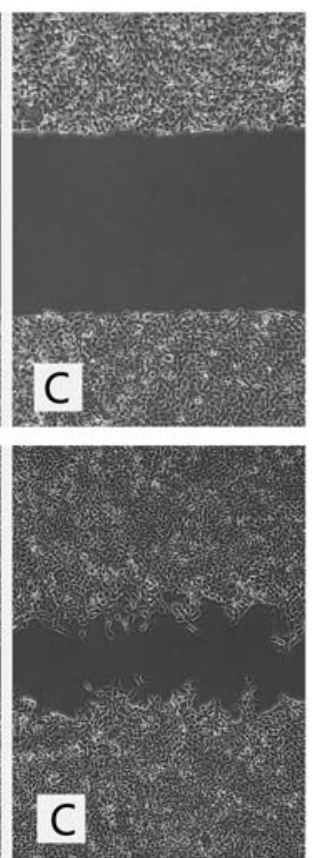

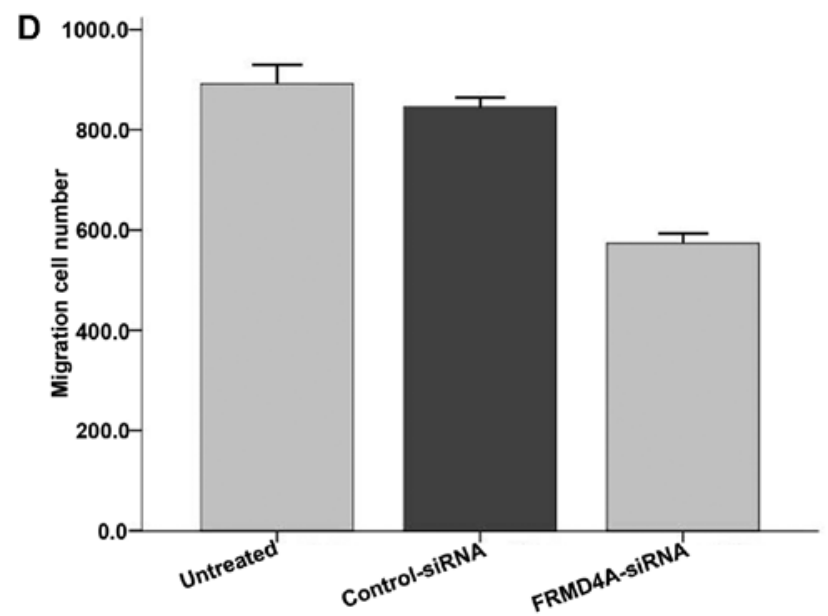

Figure 5. Analysis of cell migration. The findings of the scratch wound healing assay indicated that in the experimental group, cell migration was significantly reduced after FERM domain containing 4A (FRMD4A)-siRNA transfection. Original magnification, x100: (A) Untreated group, (B) control group, (C) experimental group. (D) Quantitative analysis of migrating cell numbers. 

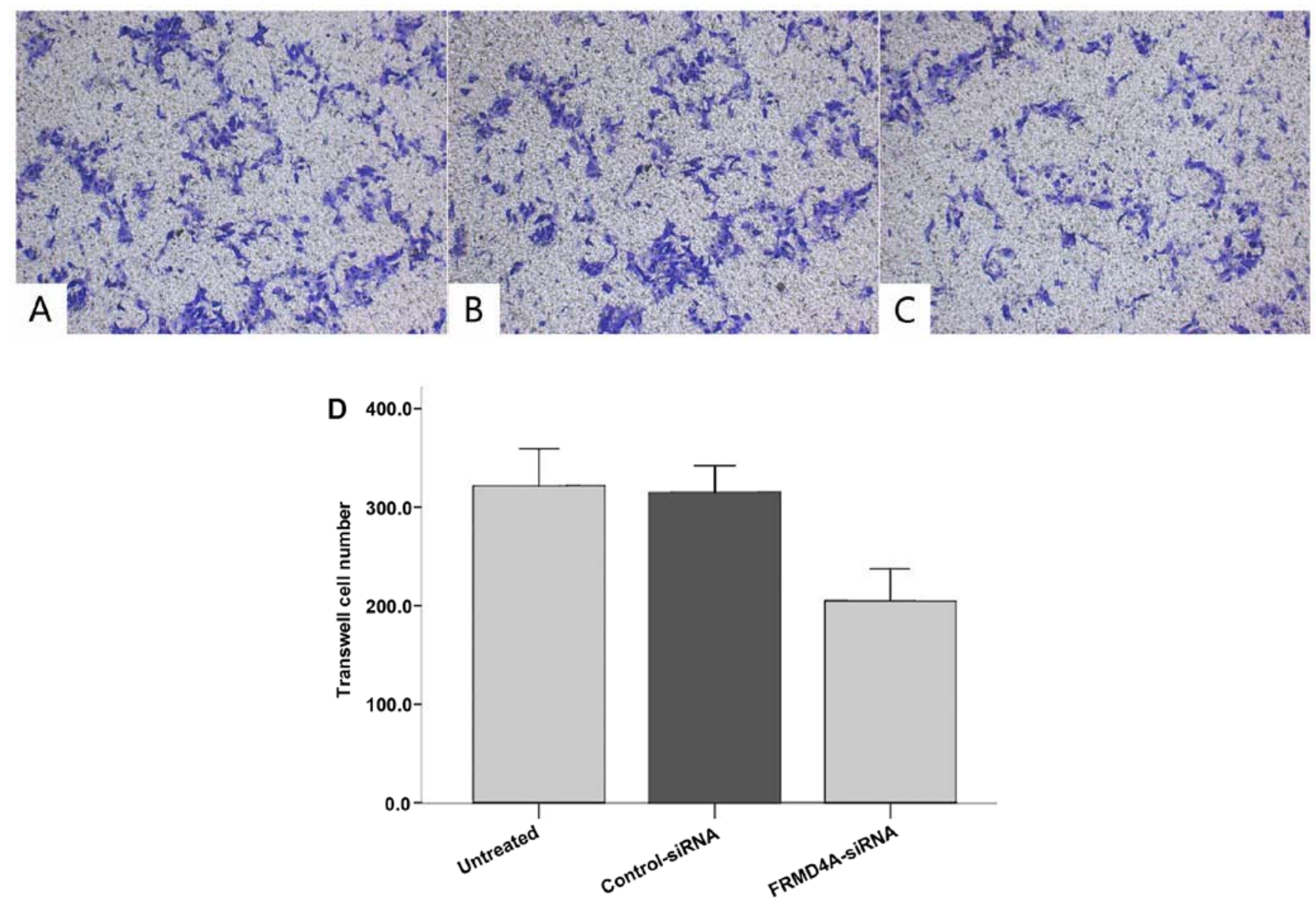

Figure 6. Analysis of cell invasion. The invasion capacity of CAL27 cells decreased in the cells transfected with FERM domain containing 4A (FRMD4A)siRNA when compared with the control groups. Original magnification, x100: (A) Untreated group, (B) control group and (C) experimental group. (D) Quantitative analysis of the number of invading cells.

transmembrane proteins to the cytoskeleton or link kinase and phosphatase enzymatic activity to the plasma membrane. FRMD4A proteins are also involved in interactions between the cell and the extracellular matrix, cell-cell communication, apoptosis, carcinogenesis and metastasis (8-11). FRMD4A was validated in a genome-wide association study in Asian populations as a gene associated with nicotine dependence in offspring and these findings were confirmed in another study $(12,13)$. The latest literature indicates that the FRMD4A gene may interact with Arf6 and the Par complex to regulate epithelial polarity (14).

In the present study, the results of immunohistochemical experiments indicated that the expression of FRMD4A protein in tongue squamous cell carcinoma was greater than that in leukoplakia or normal tissue. Positive expression was observed primarily in the cytoplasm of basal cells, and as the degree of tissue malignancy increased, it tended to spread to the spinous layer. With increases in the TNM stage and the occurrence of lymph node metastasis, the expression of FRMD4A protein level also increased, and the difference was statistically significant. However, FRMD4A expression was not associated with tumor differentiation, gender or age. Leukoplakia is a precancerous lesion (15). In this study, the expression of FRMD4A protein in leukoplakia was increased and the phenomenon of spreading to the spinous layer reflects the proliferative and regenerative capacity of the cells to some extent. For this reason, it is concluded that FRMD4A protein expression may be associated with the development of squamous cell carcinoma in the tongue. In addition, lymph node metastasis and TNM staging have been previously identified as important prognostic risk factors in patients with tongue squamous cell carcinoma $(16,17)$. Taken together, these findings suggest that FRMD4A expression is closely associated with lymph node metastasis and TNM stage, and may be indicative of a poor prognosis in patients with squamous cell carcinoma of the tongue.

When the in-depth study of tumors reached the gene level, it became feasible to identify gene targets that predict and block the proliferation and metastasis of tongue cancer and thus, reduce the mortality of patients with tongue cancer and prolong their survival. Reductions in FERM domain-containing protein activity have been studied. siRNA has been found to silence the expression of FAK and thus, to weaken the ability of AU-565 breast cancer cells (18) to migrate. The JAK inhibitor CP690 may overcome the common side effects of immunosuppressive therapy (19). RNA interference is an endogenous gene silencing mechanism. It is mediated by double stranded RNA, and it specifically inhibits the expression of homologous genes, ultimately resulting in gene silencing (20). It is highly efficient, stable and specific, and for these reasons, it has become a focus in the field of biomedical research. In this study, the FRMD4A-siRNA plasmid was transfected into 
human tongue carcinoma CAL27 cells using the liposome method, and then FMRD4A expression was evaluated using RT-qPCR and western blot analysis. The results showed that FRMD4A-siRNA successfully inhibited the expression of FRMD4A in CAL27 cells and that the inhibition rates were $94 \%$ for mRNA and $71.6 \%$ for protein.

The capacity to proliferate and to be invasive are biological characteristics of malignant tumor cells, and are also the major cause of a poor prognosis in patients (21). The results of the CCK-8 assay demonstrated that cell proliferation in the FRMD4A-siRNA group was significantly lower than that of the control groups. The G1 phase is the main control point of cell proliferation (22). FACS analysis revealed that the relative number of cells in the G1 phase was significantly higher in the FRMD4A-siRNA group than in the other groups. This suggested that G1 arrest had occurred, and that the cell cycle had slowed down. The results of the scratch wound-healing assay demonstrated that after $48 \mathrm{~h}$, the speed with which cells in the untreated group and the control-siRNA group moved to the edge of the scratch accelerated and the scratch width narrowed significantly. These two groups of cells filled the scratch area whereas the cells in the FRMD4A-siRNA group migrated more slowly. Transwell experiments showed there to be significantly fewer transmembrane cells in the FRMD4A-siRNA group than in the control-siRNA group. This indicated that silencing the gene expression of FRMD4A decreased the invasion and migration ability of CAL27 cells in vitro.

In future experiments, the research group will study the signal pathway in which FRMD4A may be involved in order to ascertain whether FRMD4A-siRNA interferes with CAL27 through direct intervention or by other means.

In conclusion, these results demonstrated that overexpression of FRMD4A occurs frequently in the process of oral carcinogenesis and that it may be closely associated with the progression of OSCCs. Silencing of the FRMD4A gene in CAL27 cells inhibited cell proliferation, led to cell cycle arrest in the G1 phase and reduced the migration and invasion capacity of CAL27 cells in vitro. Taken together, these results support the conclusion that FRMD4A may serve as a target for therapy of tongue squamous cell carcinoma.

\section{Acknowledgements}

The present study was supported by grants from the Science and Technology Program of Guangdong Province (2013B021800153) (http://www.gdstc.gov.cn/) and from the Science and Information Project Fund of Guangzhou (2014Y2-00109) (http://www.gzsi.gov.cn/).

\section{References}

1. Xiong P, Li YX, Tang YT and Chen HG: Proteomic analyses of Sirt1-mediated cisplatin resistance in OSCC cell line. Protein J 30: 499-508, 2011.
2. Ferrari D, Codeca C, Fiore J, Moneghini L, Bosari S and Foa P: Biomolecular markers in cancer of the tongue. J Oncol 2009: 412908, 2009.

3. Wu JY, Yi C, Chung HR, Wang DJ, Chang WC, Lee SY, Lin CT, Yang YC and Yang WC: Potential biomarkers in saliva for oral squamous cell carcinoma. Oral Oncol 46: 226-231, 2010.

4. Sano D and Myers JN: Metastasis of squamous cell carcinoma of the oral tongue. Cancer Metastasis Rev 26: 645-662, 2007.

5. Tepass U: FERM proteins in animal morphogenesis. Curr Opin Genet Dev 19: 357-367, 2009.

6. Yu H, Zhang Y, Ye L and Jiang WG: The FERM family proteins in cancer invasion and metastasis. Front Biosci (Landmark Ed) 16: 1536-1550, 2011.

7. Goldie SJ, Mulder KW, Tan DW, Lyons SK, Sims AH and Watt FM: FRMD4A upregulation in human squamous cell carcinoma promotes tumor growth and metastasis and is associated with poor prognosis. Cancer Res 72: 3424-3436, 2012.

8. Gastl G, Spizzo G, Obrist P, Dünser M and Mikuz G: Ep-CAM overexpression in breast cancer as a predictor of survival. Lancet 356: 1981-1982, 2000.

9. Bennett V and Baines AJ: Spectrin and ankyrin-based pathways: metazoan inventions for integrating cells into tissues. Physiol Rev 81: 1353-1392, 2001

10. Murata K, Nunomura W, Takakuwa Y and Cherr GN: Two different unique cardiac isoforms of protein $4.1 \mathrm{R}$ in zebrafish, Danio rerio, and insights into their cardiac functions as related to their unique structures. Dev Growth Differ 52: 591-602, 2010.

11. Fiévet B, Louvard D and Arpin M: ERM proteins in epithelial cell organization and functions. Biochim Biophys Acta 1773: 653-660, 2007.

12. Yoon D, Kim YJ, Cui WY, Van der Vaart A, Cho YS, Lee JY, Ma JZ, Payne TJ, Li MD and Park T: Large-scale genome-wide association study of Asian population reveals genetic factors in FRMD4A and other loci influencing smoking initiation and nicotine dependence. Hum Genet 131: 1009-1021, 2012.

13. Johnson C, Drgon T, Liu QR, Zhang PW, Walther D, Li CY, Anthony JC, Ding Y, Eaton WW and Uhl GR: Genome wide association for substance dependence: convergent results from epidemiologic and research volunteer samples. BMC Med Genet 9: 113, 2008.

14. Ikenouchi $\mathbf{J}$ and Umeda M: FRMD4A regulates epithelial polarity by connecting Arf6 activation with the PAR complex. Proc Natl Acad Sci USA 107: 748-753, 2010.

15. Kanellopoulou C and Monticelli S: A role for microRNAs in the development of the immune system and in the pathogenesis of cancer. Semin Cancer Biol 18: 79-88, 2008.

16. Bachar G, Hod R, Goldstein DP, Irish JC, Gullane PJ, Brown D, Gilbert RW, Hadar T, Feinmesser R and Shpitzer T: Outcome of oral tongue squamous cell carcinoma in patients with and without known risk factors. Oral Oncol 47: 45-50, 2011.

17. Soudry E, Preis M, Hod R, Hamzany Y, Hadar T, Bahar G, Strenov Y and Shpitzer T: Squamous cell carcinoma of the oral tongue in patients younger than 30 years: clinicopathologic features and outcome. Clin Otolaryngol 35: 307-312, 2010.

18. Earley S and Plopper GE: Disruption of focal adhesion kinase slows transendothelial migration of AU-565 breast cancer cells. Biochem Biophys Res Commun 350: 405-412, 2006.

19. Changelian PS, Flanagan ME, Ball DJ, Kent CR, Magnuson KS, Martin WH, Rizzuti BJ, Sawyer PS, Perry BD, Brissette WH, et al: Prevention of organ allograft rejection by a specific Janus kinase 3 inhibitor. Science 302: 875-878, 2003.

20. Fire A, Xu S, Montgomery MK, Kostas SA, Driver SE and Mello CC: Potent and specific genetic interference by double-stranded RNA in Caenorhabditis elegans. Nature 391: 806-811, 1998

21. An SY, Jung EJ, Lee M, Kwon TK, Sung MW, Jeon YK and Kim KH: Factors related to regional recurrence in early stage squamous cell carcinoma of the oral tongue. Clin Exp Otorhinolaryngol 1: 166-170, 2008.

22. Ho A and Dowdy SF: Regulation of G(1) cell-cycle progression by oncogenes and tumor suppressor genes. Curr Opin Genet Dev 12: 47-52, 2002. 